\title{
Der Faktor Mensch
}

\section{Monika Brodmann Maeder}

PD Dr. med. et MME, Präsidentin des SIWF

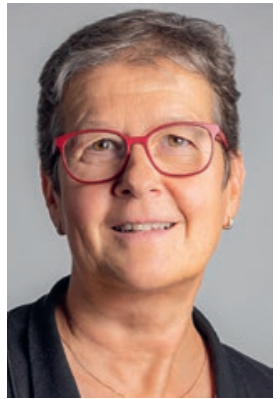

Der Ausdruck «Human Factors» tauchte erstmals in den 1940er Jahren in der Luftfahrt im Zusammenhang mit Untersuchungen von Flugunfällen in der British Royal Airforce auf. Es zeigte sich, dass viele Flugunfälle auf menschliche Unzulänglichkeiten und Fehler zurückzuführen waren und viel weniger auf technische Defekte. Die Luftfahrtindustrie reagierte auf diese Erkenntnisse mit vielfältigen Massnahmen, die vor allem das Zusammenspiel zwischen Mensch und Maschine optimieren sollten. Eine der Massnahmen betraf auch die Kommunikation im Cockpit. Das Verständnis von "Human Factors" veränderte sich und konzentrierte sich immer mehr auf das menschliche Verhalten bei der Arbeit, das sich auf die Sicherheit auswirken kann.

In den neunziger Jahren des letzten Jahrhunderts kamen spezifische Trainings auf, die sich diesen Aspekten der «Human Factors» in der Luftfahrt annahmen und den Fokus auf Teamwork und Kommunikation setzten. Bald übernahmen andere hochdynamische und risikoreiche Industrien wie die Nuklearindustrie und die Medizin diese in der Luftfahrt entwickelten Theorien von menschengemachten Fehlern. Die Pioniere waren Psychologen und Anästhesisten um David Gaba, einen Anästhesisten der Stanford University, die diese Theorien in medizinische Simulationen einbauten und die ersten CrisisRessource-Management(CRM)-Kurse für medizinische Teams entwickelten. Ein Team von Ärzten und Psycho-

\section{In der Medizin ist und bleibt der Faktor Mensch} immer noch die zentrale Essenz.

loginnen aus Schottland entwickelte ein ähnliches System für Anästhesie, Chirurgie oder Notfallmedizin und nannte es «non-technical skills» (Flin, 2011-2013). In diesen hochdynamischen Fächern der Medizin werden medizinische Simulationen als moderne Lernmethoden vermehrt eingesetzt, um diese «nicht-technischen» Fähigkeiten oder CRM-Kompetenzen der Teilnehmenden zu verbessern. Gerade die CRM-Leitsätze «Kommuniziere sicher und effektiv», "Achte auf gute Teamarbeit» oder «Setze Prioritäten dynamisch» sind gute Beispiele für die Bemühungen, die Behandlungsqualität und die Patientensicherheit zu verbessern (M. Rall, 2013). Und hier scheint es auch in der Schweiz immer noch Verbesserungspotenzial zu geben. In einer breit angelegten Umfrage des SIWF in den Jahren 2014/15 bei über 400 Weiterbildungsverantwortlichen verschiedenster Kliniken der Schweiz wurden unter anderem Defizite bei der Vermittlung von Themen wie Kommunikation, Team- und Konfliktmanagement, Leadership, Umgang mit Fehlern und $\mathrm{Pa}$ tientensicherheit festgestellt (siehe Positionspapier SIWF «Grundsätze und Empfehlungen für die Vermittlung der Allgemeinen Lernziele»).

Das Gefährlichste an der Technik ist, dass sie ablenkt von dem, was den Menschen wirklich ausmacht, von dem, was er wirklich braucht.

Elias Canetti

Die Weiterbildungsordnung des SIWF verpflichtet alle Weiterbildungsstätten zur Vermittlung dieser Themen als Allgemeine Lernziele und gibt auch Empfehlungen, wie diese vermittelt werden sollen. In den PROFILES, dem Lernzielkatalog aller medizinischer Fakultäten in der Schweiz, wird dem Thema Kommunikation viel Gewicht gegeben: Die CanMEDS-Rolle «Communicator» ist ein zentraler Punkt, der in allen neun EPAs (Entrustable Professional Activities) zu finden ist. EPA 9 mit dem Titel "Contribute to a culture of safety and improvement» widmet sich Teamaspekten oder dem Umgang mit kritischen Ereignissen und Fehlern in der Medizin. Die Grundlagen sind also sowohl in der ärztlichen Aus- als auch in der Weiter- und Fortbildung gelegt, nun müssen sie aber auch noch umgesetzt werden. Die Technik hat seit den Anfängen der «Human Factors»-Idee vor über achtzig Jahren grosse Fortschritte gemacht. Trotzdem oder gerade deshalb ist der Faktor Mensch in der Medizin immer noch die zentrale Essenz. Oder, um es mit den Worten von Elias Canetti auszudrücken: «Das Gefährlichste an der Technik ist, dass sie ablenkt von dem, was den Menschen wirklich ausmacht, von dem, was er wirklich braucht.» 